\title{
Multi-Resolution, Time-Resolved PIV Measurements of a Decelerating Turbulent Boundary Layer near Separation
}

\author{
C. Willert ${ }^{1 *}$, D. Schanz ${ }^{2}$, M. Novara ${ }^{2}$, R. Geisler ${ }^{2}$, M. Schroll ${ }^{1}$, S. Ribergård ${ }^{3}$, A. \\ Schröder ${ }^{2}$ \\ ${ }^{1}$ Institute of Propulsion Technology, German Aerospace Center (DLR), Köln, Germany \\ 2 Institute of Aerodynamics and Flow Technology, German Aerospace Center (DLR), Göttingen, Germany \\ ${ }^{3}$ Department of Mechanical Engineering, Technical University of Denmark (DTU), Lyngby, Denmark \\ *Corresponding author: chris.willert@dlr.de
}

\begin{abstract}
We report on measurements of the time-evolving velocity profile of a turbulent boundary layer subjected to a strong adverse pressure gradient (APG) at Reynolds numbers up to $R e_{\theta} \approx 55000$ with an upstream friction Reynolds number exceeding $R e_{\tau} \approx 10000$. Near the point of flow separation high-resolution imaging at high camera frame rates captured the time evolving velocity profile using the so-called "profile-PIV" technique in a nested imaging configuration of two cameras operating at different image magnifications. One camera used an image magnification better than unity to resolve the viscous scales directly at the wall while the remainder of the roughly $200 \mathrm{~mm}$ thick boundary layer is simultaneous captured by the second camera. In the APG the variance of the stream-wise velocity exhibits no "inner peak" commonly found in turbulent boundary layers without pressure gradient influence. Spectral analysis further shows that the peak energy within the boundary layer shifts away from the wall toward lower frequencies. The overlap between the simultaneously imaged areas allows to assess and, to first order, correct for the effect of spatial smoothing on statistical quantities, spectra and related quantities. A multi-frame cross-correlation algorithm was used to process the extensive data base. In addition, a newly developed 2D-2C "Shake-The-Box" algorithm (STB) provided highly resolved particle tracking data beyond the reach of conventional PIV processing.
\end{abstract}

\section{Introduction}

The evolution and characteristics of turbulent boundary layers (TBL) subjected to a positive pressure gradient are common in many flows of industrial relevance and appear, for instance, on the suction side of high-lift aircraft wing sections or on turbomachinery blading. The positive pressure gradient is associated with a deceleration of the external flow which results in a thickening of the boundary layer and reduction of the wall friction. Under strong adverse pressure gradient conditions the wall friction reduces to zero, ultimately leading to separated flow. From a numerical perspective these flows are difficult to model with methods of engineering relevance, such as Reynolds averaged Navier-Stokes simulations (RANS) and motivate experiments such as the one presented here to provide relevant validation data (see e.g. Knopp et al. (2014, 2020).

The present contribution provides an overview of the experimental facility, the design of the ramp model and focusses on the utilized measurement techniques that provide long records of time-resolved 2D $2 \mathrm{C}$ data of the TBL near the point of flow separation. The acquired data is compared with similar data obtained at the same flow conditions in the ZPG domain further upstream. The aim is to document the differences between ZPG and APG conditions at the point of flow separation, in particular, with respect to changes in the spectral properties. The presented power spectra are believed to be the first of their kind for experiments with both high Reynolds numbers and strong APG conditions at the point of separation. 


\section{Experimental setup and methods}

\subsection{Wind tunnel facility and APG model}

The measurements were performed in a Eiffel type wind tunnel with a cross section of $1.8 \mathrm{~m}$ width and $1.8 \mathrm{~m}$ height and length of $22 \mathrm{~m}$ into which a ramp model of about $7 \mathrm{~m}$ length was mounted vertically on one side of the tunnel (see Figure1). The model was designed within the DLR project VicToria and is based on a modification of a previous model design used in the DLR project RETTINA (Knopp et al., 2014) in the same facility. The design is based on 2D RANS using the DLR TAU code using the SAand SST- turbulence models with the aim of inducing flow separation along the flat section of the APG region. About $4 \mathrm{~m}$ downstream of the test section entry the flow is first accelerated by a smooth ramp of $1.7 \mathrm{~m}$ length and $0.44 \mathrm{~m}$ height and is then allowed to relax along a nearly pressure gradient free flat plate of $4 \mathrm{~m}$ length. The flow then enters a curvilinear region of $1.2 \mathrm{~m}$ length inducing a small area of favorable pressure gradient (FPG) before entering the APG domain, which consists of a flat plate of $0.76 \mathrm{~m}$ length with an inclination of about $18.6^{\circ}$ with respect to the tunnel centerline. In order to facilitate multi-camera based particle velocimetry measurements, the flat plate in the APG is fitted with a large, anti-reflection coated glass window. A similar window is present in the ZPG region to assess the incoming boundary layer. With the model mounted in an upright fashion windows on the top of the tunnel provide access for cameras viewing the plane along the centerline of the model.

In the scope of two measurement campaigns extensive measurements were performed on the configuration to globally characterize the flow using conventional PIV as well as multi-pulse STB (Novara et al. 2019). Near the point of separation 3D-STB along a $90 \mathrm{~mm}$ long cylindrical volume provided fully resolved 3D-3C data at sample rates of $20-40 \mathrm{kHz}$ (Schröder et al., 2018). The present material focuses on timeresolved profile-PIV measurements recorded in the $\mathrm{ZPG}$ domain at $X=-3730 \mathrm{~mm}\left(X_{T}=7.65 \mathrm{~m}\right)$ and in the APG at $X=-620 \mathrm{~mm}\left(X_{T}=10.76 \mathrm{~m}\right)$ which is in the immediate vicinity of the mean point of flow separation. Here, $X_{T}$ is referenced to the entry of the test section, while $X=0$ coincides with the downstream end of the ramp model.

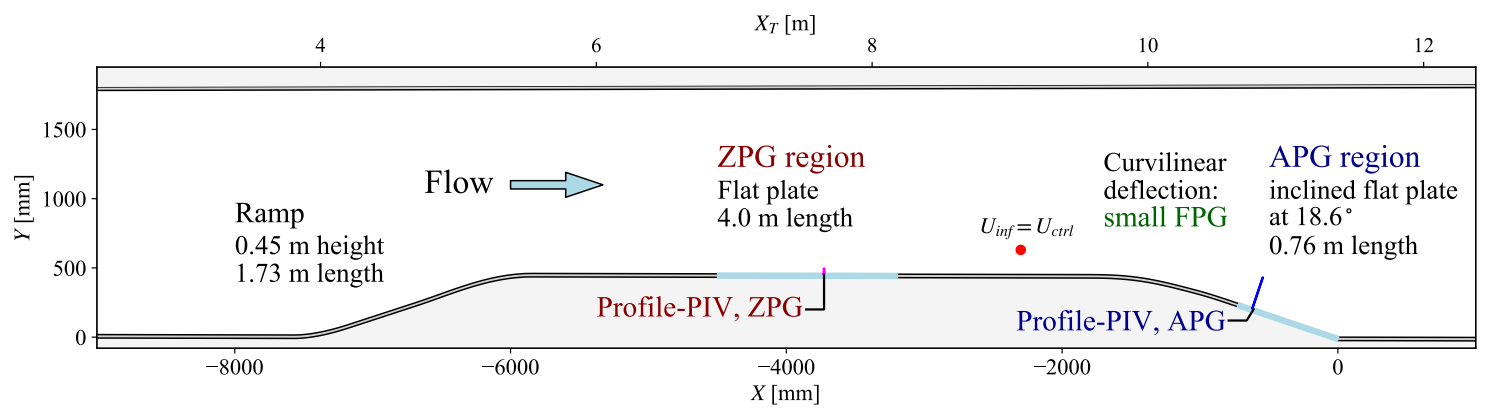

Figure 1: Schematic of the ramp model within the wind tunnel test section with indication of the measurement locations for the profile-PIV technique.

\subsection{Particle imaging methods for time-resolved profile measurements}

During the measurement campaigns a variety of PIV and Lagrangian particle tracking (LPT) techniques provided an extensive data base of the flow field extending from the ZPG domain well into the APG area. An overview is provided in Schröder et al. (2018). The present contribution focusses on time-resolved 2D $2 \mathrm{C}$ measurement configurations that provided time-resolved velocity profiles at selected positions.

All particle image methods relied on aerosol seeding based on $\approx 1 \mu \mathrm{m}$ DEHS droplets which was supplied upstream of the entry to the test section through several Laskin-type atomizing seeding generators. Both near-wall and global seeding was used. The large travelling distance of nearly $10 \mathrm{~m}$ to the measurement location ensured a homogenous distribution of particles throughout the TBL.

\subsubsection{High-speed 2D2C PIV imaging}

For the profile-PIV measurements in the ZPG region $(X=-3730 \mathrm{~mm})$ the PIV system was operated with a Photron SA-X2 high speed camera with $20 \mu \mathrm{m}$ pixel pitch equipped with a $200 \mathrm{~mm}$ f/4 lens (Nikon Micro 
Nikkor 200/4) coupled to a 2x-teleconverter (Nikon TC2.0E). Depending on the flow velocity the camera was operated at frame rates between $30-50 \mathrm{kHz}$ in a reduced resolution mode of $152 \times 1024$ pixels which corresponds to a physical FOV of $6.6 \times 45 \mathrm{~mm}^{2}$ in $x$ - and $y$-direction and image magnification of $\approx 23 \mathrm{px} / \mathrm{mm}$ $(\approx 43 \mu \mathrm{m} / \mathrm{px})$. Illumination was provided in a focused beam of $6 \mathrm{~mm}$ extension in streamwise direction and $0.8 \mathrm{~mm}$ (spanwise) thickness by a high repetition rate Q-switched laser (Innolas Photonics, Blizz 30) with $38 \mathrm{~W}$ total power at 30 to $50 \mathrm{kHz}$. In order to reduce pixel locking on the rather large sensor pixels, an optical diffuser filter (LaVision) was placed in front of the sensor. For each of the $U_{e}$ velocities, two long time-resolved sequences of 294,000 images each were acquired.

\subsubsection{Nested high-speed 2D2C PIV imaging}

In the APG region the boundary layer was simultaneously imaged at two magnifications using two cameras (Vision Research V2640) each with 144 GB of on-board memory and a detector array size of $2048 \times 1952$ pixels with a square pitch of $13.5 \mu \mathrm{m}$ per pixel. To operate the cameras at a frame rate of $50 \mathrm{kHz}$ the active pixel area was reduced to $224(X) \times 2048(Y)$ pixel. While one camera (Camera 1) operated at a magnification of better than unity to capture only the near wall flow, the second camera (Camera 2) captured the entire extent of the boundary layer of approximately $200 \mathrm{~mm}$ thickness. The high image magnification at a working distance of about $950 \mathrm{~mm}$ for Camera 1 could be achieved with a $300 \mathrm{~mm} \mathrm{f} / 4$ lens (Nikon 300 F4 IF-ED AF) combined with a $2 x$ teleconverter (Nikon TC2.0E) and an extension tube of about $100 \mathrm{~mm}$ length (see Fig. 2 , middle). Camera 2 used a $100 \mathrm{~mm}$ f/2 lens (Zeiss Macro Planar T 100/2.0 ZF) to image a field of view of $250 \mathrm{~mm}$ height. A mirror placed in front of Camera 2 was necessary to align the viewing axes parallel to each other as the camera cases were significantly larger than the required $\approx 100 \mathrm{~mm}$ separation between the two cameras (see Fig. 2, right).

The narrow field of view was illuminated by a double cavity high-speed laser (Photonics Industries) with a maximum integral power of $2 \times 150 \mathrm{~W}$ capable of independently operating at up to $50 \mathrm{kHz}$. Its combined beam was spread to about $15 \mathrm{~mm}$ width and $1-2 \mathrm{~mm}$ thickness across the field of view owing to laser beam's divergence.

Measurement data was acquired for three free stream velocities either as contiguous long sequences of about $4.4 \mathrm{~s}$ length $(220,000$ frames $)$ or as individual bursts of 10,000 frames each $(0.2 \mathrm{~s})$. In burst-mode, 300 uncorrelated samples were acquired for each free-stream condition to ensure statistical convergence. A minimum of 10 long records of 220,000 frames for each condition completes the raw image data base for the APG area, totaling about $24 \mathrm{~TB}$ in size $\left(\approx 2.2 \times 10^{7}\right.$ images $)$.
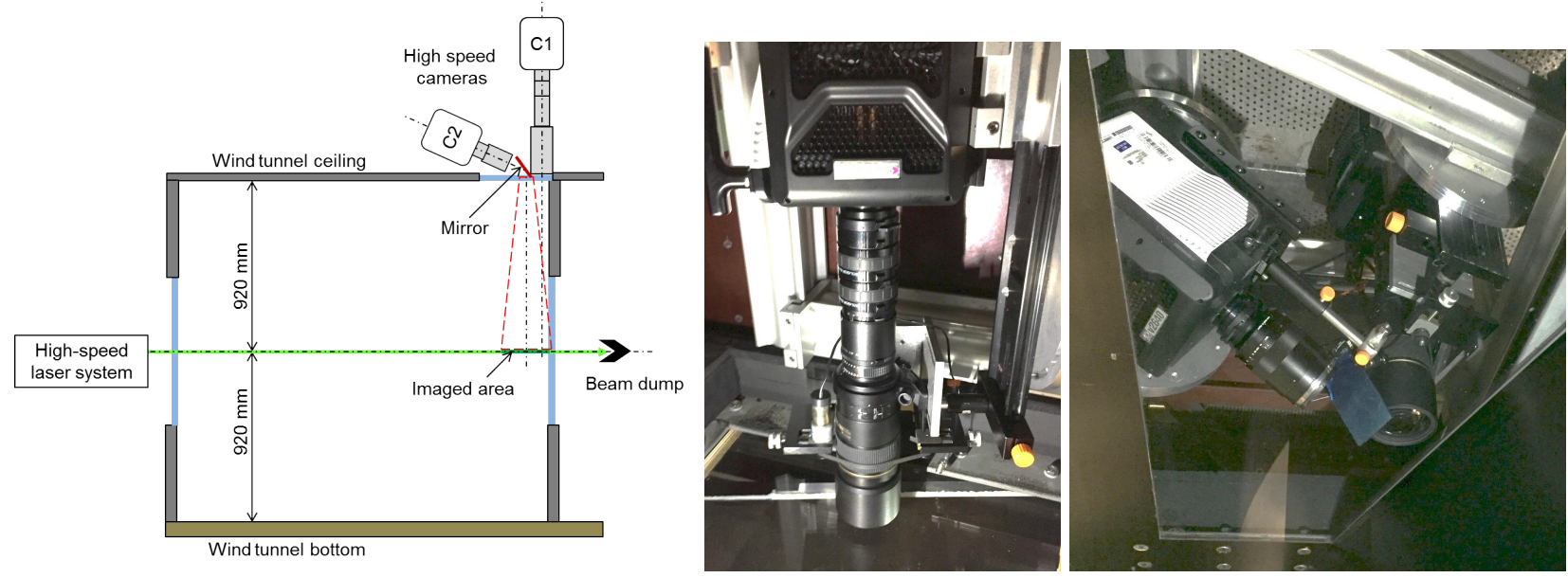

Figure 2: Left: Diagram of nested PIV imaging setup in the APG with camera 1 operating at $M=1.1$ and camera 2 operating at $M=0.11$; middle: photograph of installed camera 1 with $600 \mathrm{~mm}$ focal length imaging configuration; right: installation of both cameras above the wind tunnel as seen from the inside of the test section. 


\section{Data processing}

\subsection{PIV processing}

The acquired 2D 2C PIV image data was processed using a combination of in-house and commercial software (PIVview v3.8, PIVTEC GmbH) augmented with Python-based batch processing and post-processing tools. To achieve optimal, noise-minimized velocity estimates, a pyramid correlation scheme, similar to an algorithm proposed by Lynch and Scarano (2013), was employed and used up to 7 consecutive frames. Shown in Fig. 3, left, the algorithm makes use of the fact that the images are uniformly spaced in time with increments of $\Delta t$. This allows the calculation of cross-correlation on image pairs spaced at uniform intervals $n \Delta t$ with $1<n<N_{\max }$. Results from the pair-wise correlation are combined in a weighted manner, either through summation of correlation planes or by averaging of the individual displacement estimates. Underlying the correlation process is a coarse-to-fine scheme including sub-pixel image-shifting. Iteratively, the images are thereby shifted toward the central image of the image sub-set, such that the correlation peaks move toward the origin and the residual offset between samples is minimized. Due to the averaging of correlation planes noise peaks are attenuated.

The processing time of the multi-frame processing scheme with respect to the number of sampled frames $N$ is presented in Fig. 3 b. In comparison to dual-frame analysis, the 3 -frame scheme requires roughly $30 \%$ more time which can be explained by the fact that the computationally heavy sub-pixel image shifting only needs to be applied on two of the three images. The additional correlations can be efficiently implemented by computing forward Fourier transforms only once for each sample for re-use at different image strides. In spite of the algorithm's overall efficiency, the large amount of raw image data (ca. 25 TB for APG and ZPG combined) was processed with triple-frame sub-samples rather than the more accurate five-frame approach. Using the above multi-framing processing strategy required about two months on a state-of-theart workstation with 16-core CPU (AMD Ryzen Threadripper 2950X, 16 cores, 3.5 GHz, 64GB RAM).

The difference in magnification leads to a attenuation of fine-scale structures of camera 2 vs. camera 1. This leads to an underestimation of the velocity variances for camera 2. To first order, this can be compensated through the availability of data seen by both cameras and has been applied to correct the variance data shown in Fig. 6d).
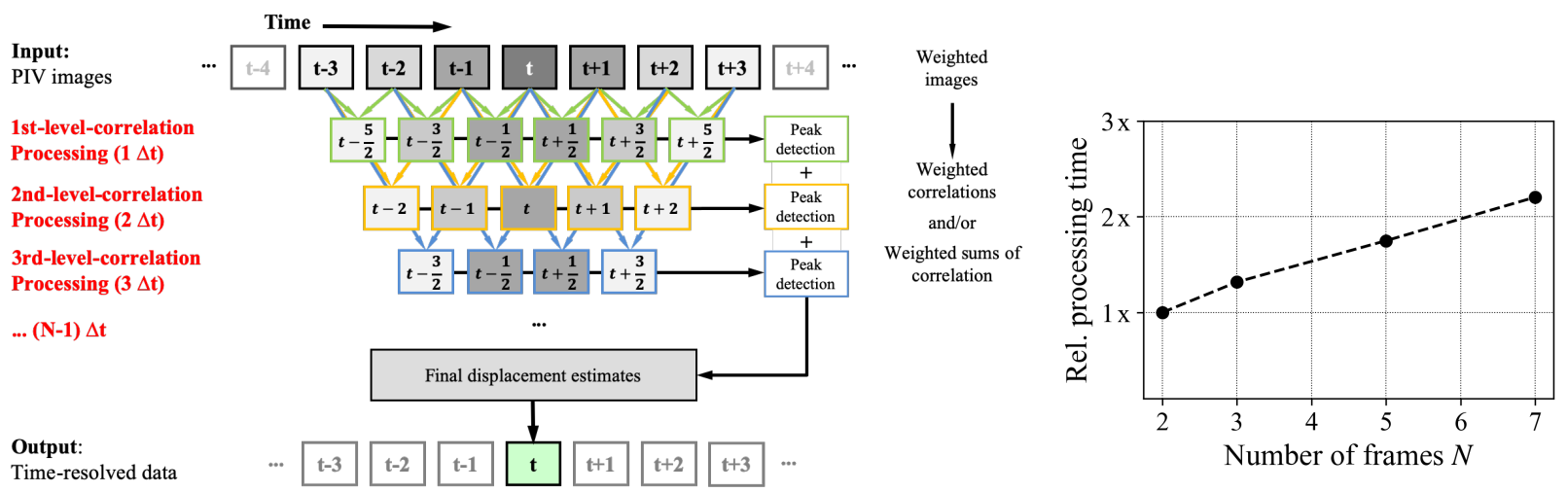

Figure 3: Left: schematic of the utilized pyramid correlation scheme, here for 7 consecutive images. Right: processing speed with respect to standard two-frame cross-correlation analysis.

\subsection{D-STB processing}

The image data was also processed using a 2D-2C-PTV algorithm, derived from the 3D-3C "Shake-TheBox" (STB) Lagrangian particle tracking (LPT) method by Schanz et al. (2016). For this purpose, the STB predictor/corrector-scheme was adapted to allow for the tracking of particles on time-resolved recordings based on single camera views, yielding time-resolved 2D-2C velocity data along tracks. The new 2D-STB evaluation scheme is based on the core functionality of STB involving particle position prediction followed by correcting for the introduced error by 'shaking' the predicted particle position (similar to IPR, Wieneke 2012). However, the volume reconstruction is replaced by a simple peak search on the 2D image. The method works reliably in finding tracks in images with moderate particle image densities. The method is 
Table 1: Profile-PIV imaging and processing parameters

\begin{tabular}{llllll}
\hline Condition & & ZPG & $\begin{array}{l}\text { APG } \\
\text { Camera 1 }\end{array}$ & $\begin{array}{l}\text { APG } \\
\text { Camera 2 }\end{array}$ & \\
\hline Magnification & $\mathrm{m}$ & 0.464 & 1.10 & 0.111 & \\
& $\mathrm{~m}$ & 43.1 & 12.3 & 122 & {$[\mu \mathrm{m} /$ pixel] } \\
Pixel size & & 20 & 13.5 & 13.5 & {$[\mu \mathrm{m}]$} \\
Field of view & $X \times Y$ & $152 \times 1024$ & $224 \times 2048$ & $224 \times 2048$ & {$[\mathrm{pixel]}$} \\
& $X \times Y$ & $6.55 \times 44.1$ & $2.75 \times 25.2$ & $27.3 \times 249.8$ & {$\left[\mathrm{~mm}^{2}\right]$} \\
PIV sampling window & $X \times Y$ & $64 \times 3$ & $32 \times 12$ & $24 \times 12$ & {$[\mathrm{pixel]}$} \\
& $X \times Y$ & $2.76 \times 0.129$ & $0.393 \times 0.147$ & $2.93 \times 1.46$ & {$\left[\mathrm{~mm}^{2}\right]$} \\
PIV grid spacing & $X \times Y$ & $24 \times 1$ & $16 \times 4$ & $8 \times 4$ & {$[\mathrm{pixel]}$} \\
& $X \times Y$ & $1.03 \times 0.043$ & $0.197 \times 0.049$ & $0.976 \times 0.488$ & {$\left[\mathrm{~mm}^{2}\right]$} \\
\hline
\end{tabular}

computationally efficient and scales with the number of tracked particles. For the present data the newly developed STB evaluation is able to identify and follow more than 2,200 particle tracks per time-step. An evaluation based on a fraction of the complete data set already shows the performance gain resulting in a much higher spatial resolution compared to the non-isotropic cross-correlation approach. To calculate the velocity statistics a bin-averaging scheme with bin-heights of 0.25 pixels $\left(<1^{+}\right)$in wall normal direction was used and results in about 100,000 entries per bin for one of three available runs. The limiting factor for fully converged statistics of such a time-resolved particle tracking measurement are the temporal scales or turn-over eddy times of superstructures embedded in the outer logarithmic region of the TBL flow $(\approx 0.4 \delta)$, requiring a multitude of short (uncorrelated) records or very long image sequences covering many eddy turn-over times.

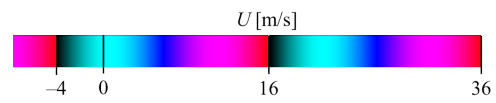

Camera 2

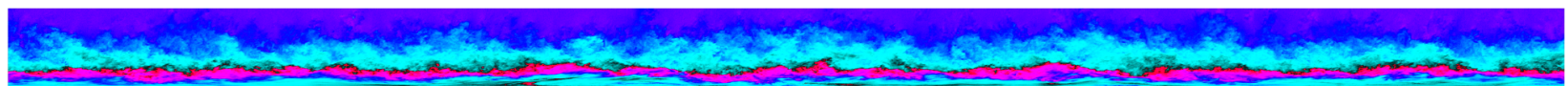

Camera 1

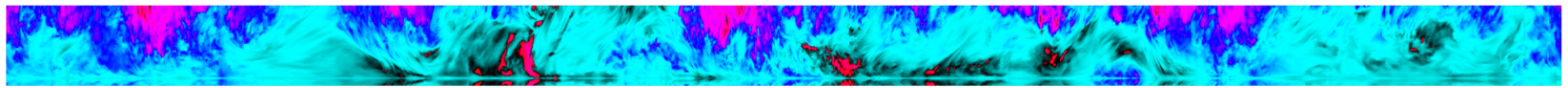

Figure 4: Time-records covering $0.2 \mathrm{~s}$ (10,000 samples) of the stream-wise velocity profile $U$ for camera 2 (top) spanning a wall-normal distance of $y=245 \mathrm{~mm}$ and camera 1 (bottom) spanning $y=24.5 \mathrm{~mm}$ at $U_{\infty}=28.7 \mathrm{~m} / \mathrm{s}$.

\section{Results}

\subsection{Velocity profile statistics}

Most of the data post-processing is based on time-records of a single column of data extracted from the center of the PIV processing domain, resulting in space-time records. An example of such space-time records visualizing the streamwise velocity component $U$ simultaneously acquired by both cameras is shown in Fig. 4. A detail of this figure is provided in Fig. 5f for the high resolution camera 1. The colorbar is chosen such that strong upstream flow near the wall is highlighted as red-purple patches representing values on the order $20 \%$ of $U_{e}$. On the whole, the highly dynamic near-wall flow fluctuates around a mean near zero (cyan color) which is due to the APG induced deceleration of the external flow, that is, the flow is on the verge of separation.

The strong flow reversal in Fig. 5 extend far beyond the viscous region. Topologically these flow reversal features differ significantly from those previously observed under ZPG and mild APG conditions in which 


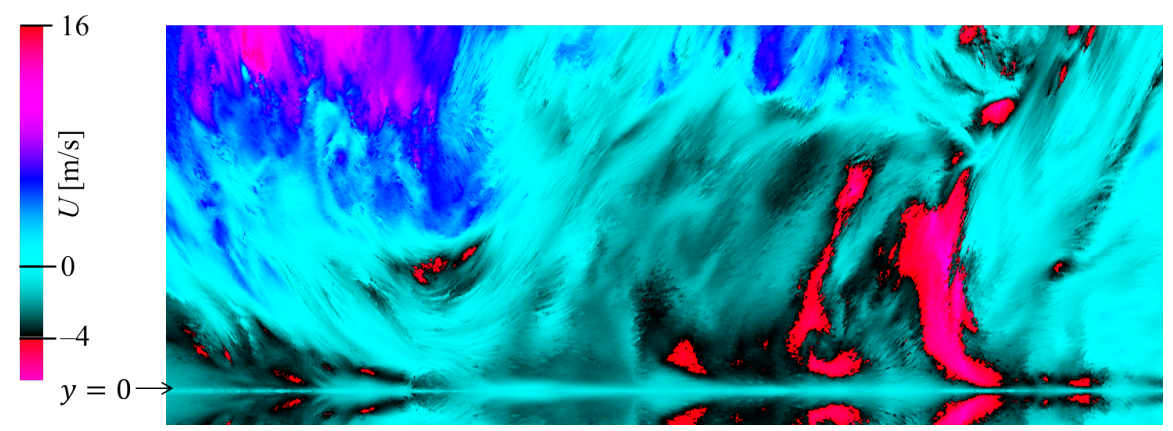

Figure 5: Detail of the time-evolving streamwise velocity profile obtained from high-magnification camera 1 covering 1250 samples $(0.025 \mathrm{~s})$ at $U_{\infty}=28.7 \mathrm{~m} / \mathrm{s}$. The vertical axis spans $y \approx 24 \mathrm{~mm}$.

Table 2: Boundary layer parameters for different wind tunnel operating conditions

\begin{tabular}{llllllll}
\hline Condition & & ZPG & & APG & & \\
Upstream velocity & $\mathbf{U}_{\infty}$ & $\mathbf{2 9 . 0}$ & $\mathbf{3 5 . 0}$ & $\mathbf{2 8 . 7}$ & $\mathbf{3 5 . 2}$ & $\mathbf{4 1 . 8}$ & {$[\mathrm{m} / \mathrm{s}]$} \\
\hline Edge velocity & $U_{e}$ & 29.0 & 35.0 & 22.3 & 27.3 & 32.1 & {$[\mathrm{~m} / \mathrm{s}]$} \\
Boundary layer thickness & $\delta_{99}$ & 80.0 & 79.0 & 207.8 & 205.0 & 201.3 & {$[\mathrm{~mm}]$} \\
Displacement thickness & $\delta^{*}$ & 9.16 & 9.05 & 61.3 & 57.0 & 51.8 & {$[\mathrm{~mm}]$} \\
Momentum thickness & $\theta$ & 7.05 & 7.01 & 27.1 & 26.5 & 25.5 & {$[\mathrm{~mm}]$} \\
Shape factor & $H=\delta^{*} / \theta$ & 1.30 & 1.29 & 2.35 & 2.15 & 2.03 & \\
Reynolds number & $\operatorname{Re}_{\theta}=U_{e} \theta / \mathrm{v}$ & 12,200 & 14,700 & 40,000 & 48,000 & 55,000 & \\
Friction velocity & $u_{\tau}$ & 1.030 & 1.225 & n.a. & n.a. & n.a. & {$[\mathrm{m} / \mathrm{s}]$} \\
Viscous unit & $v / u_{\tau}$ & 16.4 & 13.7 & n.a. & n.a. & n.a. & {$[\mu \mathrm{m}]$} \\
Estimated wall shear & $d u / d y_{y=0}$ & 63800 & 89700 & 150 & 1050 & 4000 & {$\left[\mathrm{~s}^{-1}\right]$} \\
\hline
\end{tabular}

they are both confined to the viscous layer at $y^{+} \leq 5$ and much more short-lived (Lenaers et al., 2012; Willert et al., 2018a b). The underlying mechanisms of the flow reversal phenomena observed here are believed to be completely different and unrelated to those previously observed.

Mean velocity profiles for several free stream conditions are provided in Fig. 6a and 6b, normalized by the respective edge velocities $U_{e}$ and boundary layer thickness $\delta_{99}$. While the profiles for ZPG are representative for this type of flow, the APG flow condition exhibits no clear logarithmic region such that an estimation of viscous scales based on a Clauser fit (Clauser, 1954) or based on the wall-shear stress is not straight forward and possibly not even adequate as the mean wall-shear stress approaches zero. For this reason and in order to compare the APG flow with that of ZPG, all data have been normalized by the boundary layer thickness $\delta_{99}$ and local free stream velocity $U_{e}$.

Fig. 6e and 6d show profiles of the variances $\left\langle u^{\prime} u^{\prime}\right\rangle,\left\langle v^{\prime} v^{\prime}\right\rangle$ and the associated Reynolds shear stress $\left\langle u^{\prime} v^{\prime}\right\rangle$ for the two pressure gradient conditions. Noteworthy is that the near-wall high-intensity turbulence or "inner peak" commonly found for ZPG or mild APG conditions (c.f. Fig.6d) is completely attenuated in the present APG condition. Remnants of the inner peak can be observed in the range $\left(0.001<y / \delta_{99}<0.01\right)$. Instead, the streamwise turbulence peaks at about $0.15 \delta_{99}$, placing it several hundred viscous units away from the wall, assuming viscous units on the order of $20-50 \mu \mathrm{m}$ (the actual value has yet to be determined).

The APG velocity profiles shown in Fig. 6p show a very weak gradient near the wall which is highlighted more clearly by plots of the mean velocity gradient $d U / d y$ provided in Fig. 7. In comparison to the ZPG TBL (Fig. 7 $\mathrm{a}$ ), where the highest velocity gradient is present in the immediate vicinity of the wall and rapidly decays with increased wall distance, the APG TBL exhibits a plateau of nearly constant shear rate in the range $\left(0.01<y / \delta_{99}<0.02\right)$, also peaking at about $0.15 \delta_{99}$ coinciding with the location of the peak streamwise turbulence. Beyond $0.4 \delta_{99}$ the velocity gradient decays linearly toward zero at the outer edge of the TBL. To more clearly highlight the difference between ZPG and APG plots of the velocity gradient with linear axis scaling are also shown in Fig.7. 


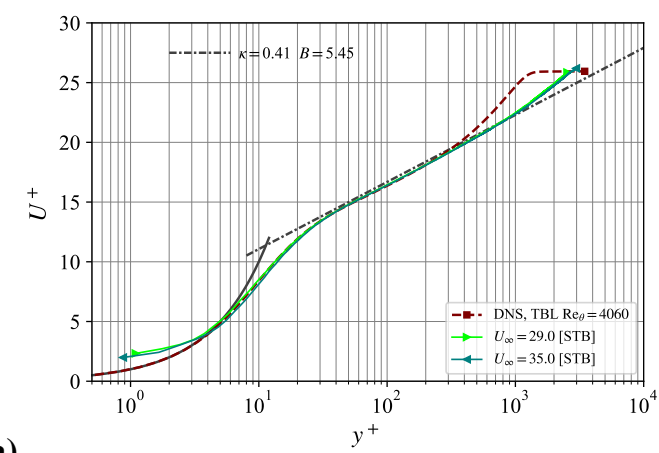

a)

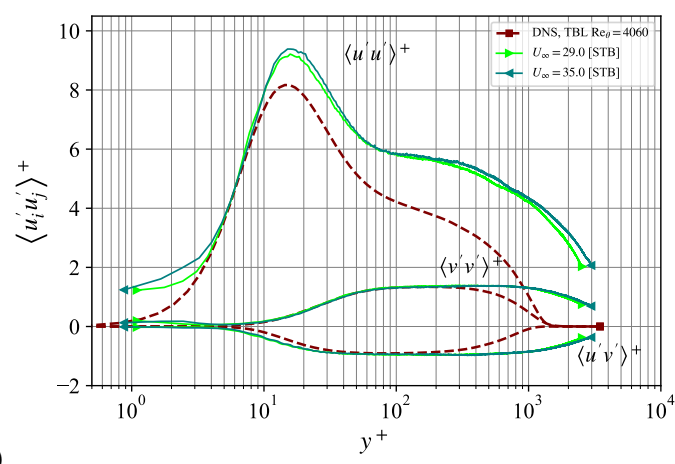

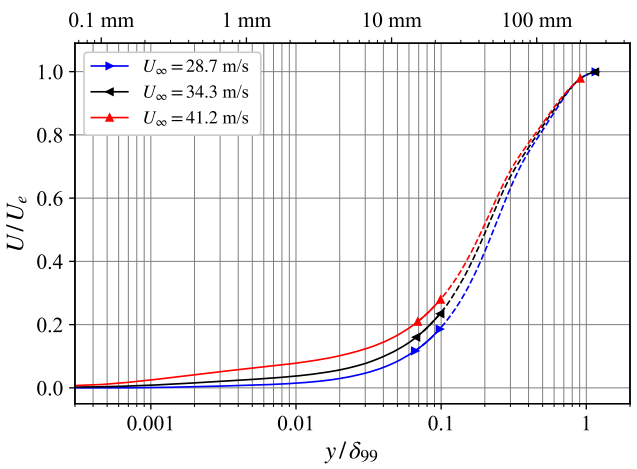

b)

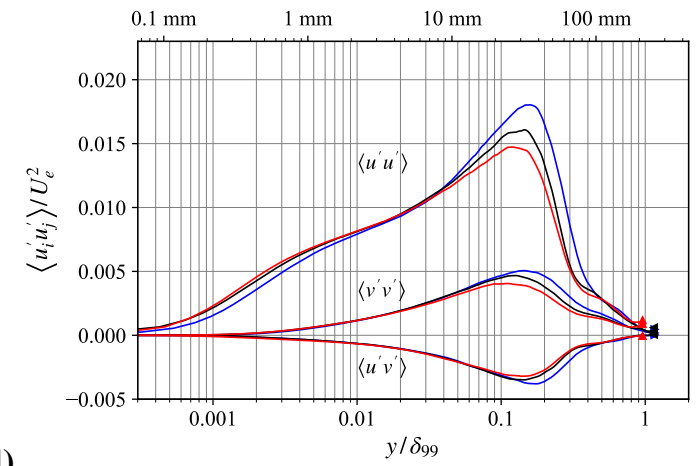

Figure 6: Mean streamwise velocity profiles (a,b) and variances and covariance of the streamwise and wall-normal velocity components $(\mathrm{c}, \mathrm{d})$, for ZPG condition $(\mathrm{a}, \mathrm{c})$ und APG $(\mathrm{b}, \mathrm{d})$. All profiles are scaled with respective edge velocities $U_{e}$ and boundary layer thicknesses $\delta_{99}$. Solid lines in (b) are obtained from high-magnification camera 1 whereas dashed lines are associated with full-view camera 2.

\subsection{Velocity spectra}

The good temporal resolution and long record lengths permit the estimation of power spectral density distribution across the entire vertical extent of the field of view. To illustrate the construction of the 2D spectral maps from the velocity time records, a sample spectra for a fixed wall distance is provided in Fig. 8 (left) for the stream-wise velocity $U$ and wall-normal velocity $V$. The $2 \mathrm{D}$ contour map shown in Fig. 8 (right) is assembled by plotting the individual spectra for all wall distances $y$. The discontinuity in the spectra at $y=20 \mathrm{~mm}$ is due to spatial filtering of camera 2 with respect to camera 1 . The spatial filtering results in the attenuation of the smaller scales which typically are also associated with reduced time scales.

In the following, spatially resolved spectra obtained in the ZPG and APG are presented side-by-side for matching flow conditions at $U_{\infty}=29.0 \mathrm{~m} / \mathrm{s}$. It should be noted that the spectra are pre-multiplied by the frequency in order to better highlight the prominent features. Due to spatial resolution limits in the ZPG measurements, attenuation is present close to the wall for $y^{+}<20$ (less than $\approx 0.2 \mathrm{~mm}$ ). This leads to an attenuation of the spectral peak normally found at the position of the inner turbulence peak at $y^{+} \approx 15$. Nonetheless, the inner peak can be clearly identified in Fig. $9 \mathrm{a}$ at reduced frequency near one with its wallnormal position indicated by the dashed black line. Under the present APG conditions, at the point of flow separation, the peak energy shifts away from the wall to about $10 \%$ of the boundary layer thickness (Fig. 9p). A near-wall energy peak can no longer be identified. Also the peak energy shifts towards an order of magnitude lower frequency.

For the energy spectra of the wall-normal fluctuations, shown in Fig. $9 \mathrm{k}$ and $9 \mathrm{~d}$, the shift of peak energy content is not as pronounced in its spatial movement with its highest energy also shifting to $0.15 y / \delta$. However, under APG conditions the reduced frequency of the wall-normal velocity shifts to a peak value centers close to unity. The dominant frequency indicates a periodic vertical motion at a timescale corresponding to the eddy turnover time $\delta / U_{e}$ and may be interpreted as vortex shedding process in the free shear layer that begins to form as the flow undergoes separation.

The spectral energy distribution of the Reynolds stress $\left\langle u^{\prime} v^{\prime}\right\rangle$ is provided in Fig. 9e and 9F. For the ZPG condition, the distribution is in agreement with DNS data reported in the literature (see e.g. Âhn et al. 2015) 

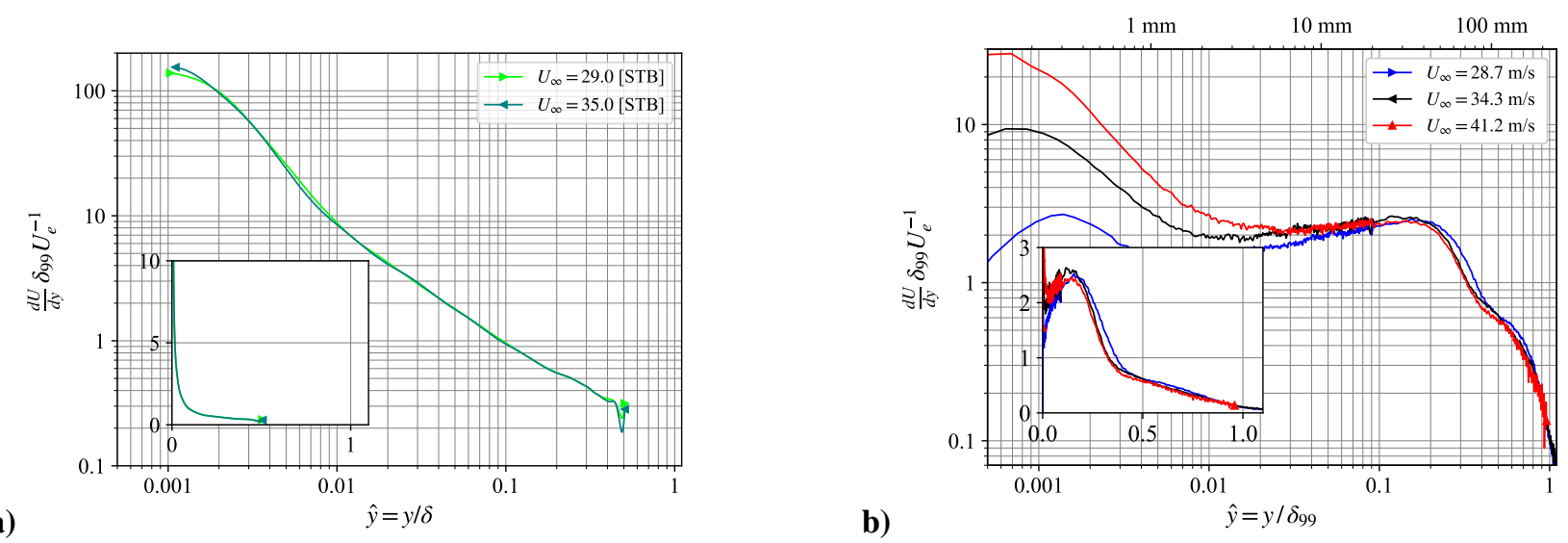

Figure 7: Mean velocity gradient $d U / d y$ for ZPG condition (a) and APG (b) normalized by boundary layer thickness $\delta_{99}$ and edge velocity $U_{e}$. Inserts show the same data with linear axis scaling.

Table 3: Spectral peak positions as indicated by white stars in Fig.9.

\begin{tabular}{|cllll|llll|c|}
\hline \multicolumn{2}{|l}{ ZPG } & & & & APG & & & & Ratio \\
& $y$ & $y / \delta_{99}$ & $f$ & $\hat{f}$ & $y$ & $y / \delta_{99}$ & $f$ & $\hat{f}$ & $\hat{f}_{\mathrm{ZPG}} / \hat{f}_{\text {APG }}$ \\
\hline$\left\langle u^{\prime} u^{\prime}\right\rangle$ & $0.22 \mathrm{~mm}$ & 0.0027 & $490 \mathrm{~Hz}$ & 1.35 & $34.3 \mathrm{~mm}$ & 0.152 & $30.5 \mathrm{~Hz}$ & 0.29 & 4.7 \\
$\left\langle v^{\prime} v^{\prime}\right\rangle$ & $3.62 \mathrm{~mm}$ & 0.045 & $1410 \mathrm{~Hz}$ & 3.88 & $33.3 \mathrm{~mm}$ & 0.145 & $122 \mathrm{~Hz}$ & 1.15 & 3.4 \\
$\left\langle u^{\prime} v^{\prime}\right\rangle$ & $0.56 \mathrm{~mm}$ & 0.007 & $1074 \mathrm{~Hz}$ & 2.97 & $34.4 \mathrm{~mm}$ & 0.150 & $48.8 \mathrm{~Hz}$ & 0.46 & 6.5 \\
\hline
\end{tabular}

with a broad distribution throughout the extent of the boundary layer. Under APG conditions the Reynolds stress is concentrated near $0.15 y / \delta$, similar to the streamwise and wall-normal fluctuations.

Aside from the movement of peak energy away from the wall, there is also a shift toward lower frequencies by about one order of magnitude as the flow reaches the APG. When normalized against the increased boundary layer thickness $\delta_{99}$ and decreased edge velocity $U_{e}$ the reduction is less significant with the ratio changing from $U_{e} /\left.\delta_{99}\right|_{\mathrm{ZPG}}=363 \mathrm{~Hz}$ to $U_{e} /\left.\delta_{99}\right|_{\mathrm{APG}}=108 \mathrm{~Hz}$ by a factor of $\approx 3.4$, which is of similar magnitude as the change in normalized frequency (c.f. Table 3). The frequency change can thus be explained by the strong thickening of the boundary layer along with its deceleration. The low frequency content is indicative of the formation of large scale (outer) structures as observed in large FOV measurements.

\section{Conclusions}

Highly resolved velocity data in both space and time were acquired in a high Reynolds number turbulent boundary layer subjected to strong adverse pressure gradient conditions. A nested camera imaging configuration was chosen in order to capture both the near-wall dynamics and at the same time have access to velocity data throughout the entire boundary layer height. The acquired data was compared to similar profile data acquired in the near-zero pressure gradient zone upstream of the APG domain. At the point of separation the mean velocity gradient exhibits a region of uniform shear peaking at about $15 \%$ of the TBL thickness, the position at which both the velocity variances and Reynolds stress have their maximum intensity and highest spectral intensity. The extensive data base still offers access to further quantities and associated analysis, such as the interaction of the unsteady wall-shear with the outer flow.

On a general note, the data provided by the profile-PIV technique is well suited for analysis methods commonly used by single-point measurements such as HWA and LDA, but additionally offers a spacetime coherence across the field of view, providing possibilities for more advanced analysis yet to be fully explored. In a next post-processing step it is foreseen to compare a B-spline based 2D interpolation scheme (similar to FlowFit (Gesemann et al., 2016) applied to the scattered 2D-STB velocity and acceleration data of the particle tracks with the correlation based velocity fields from profile-PIV. 


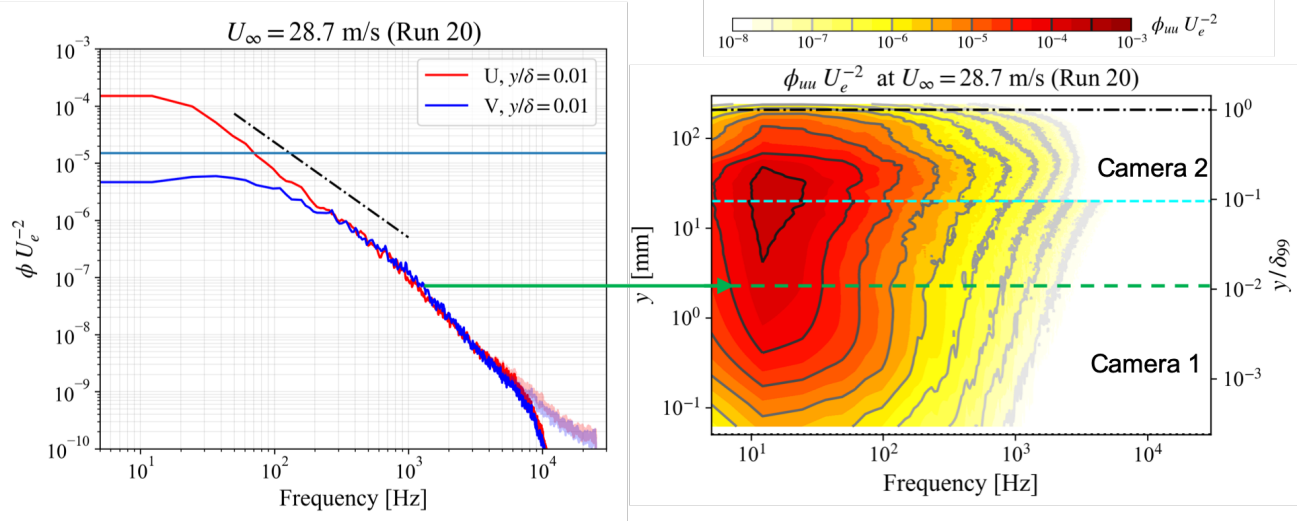

Figure 8: Power spectra obtained for the streamwise velocity component. Highlighted curves in 1-D spectra (left) are lowpass filtered at $10 \mathrm{kHz}$ of the original signal shown in faded colors. Solid blue line in left subfigure represents a rms fluctuation of 1 pixel. Dashed light-blue line in right subfigure indicates a chosen boundary at $y=15 \mathrm{~mm}$ between camera view 1 and view 2.

\section{Acknowledgements}

The authors would like to thank Prof. Christian Kähler and his team for their support during the measurement campaigns and the use of the wind tunnel facility along with the high-power pulsed laser system.

\section{References}

Ahn J, Lee JH, Lee J, Kang Jh, and Sung HJ (2015) Direct numerical simulation of a 30R long turbulent pipe flow at $R e_{\tau}=3008$. Physics of Fluids 27:065110

Clauser FH (1954) Turbulent boundary layers in adverse pressure gradients. Journal of the Aeronautical Sciences 21:91-108

Gesemann S, Huhn F, Schanz D, and Schröder A (2016) From noisy particle tracks to velocity, acceleration and pressure fields using b-splines and penalties. in 18th International Symposium on Applications of Laser Techniques to Fluid Mechanics. number 186 in Conference Proceedings online, Book of Abstracts. pages 1-17. paper 04.5_4_186

Knopp T, Novara M, Schanz D, Geisler R, Philipp F, Schroll M, Willert C, and Schröder A (2020) Modification of the SSG/LRR- $\omega$ RSM for turbulent boundary layers at adverse pressure gradient with separation using the new DLR VicToria experiment. in A Dillmann, G Heller, E Krämer, C Wagner, C Tropea, and S Jakirlić, editors, New Results in Numerical and Experimental Fluid Mechanics XII. pages 80-89. Springer International Publishing, Cham

Knopp T, Schanz D, Schröder A, Dumitra M, Hain R, and Kähler CJ (2014) Experimental investigation of the log-law for an adverse pressure gradient turbulent boundary layer flow at $\operatorname{Re}_{\theta}$ up to 10000. Flow Turbulence and Combustion 92:451-471

Lenaers P, Li Q, Brethouwer G, Schlatter P, and Örlü R (2012) Rare backflow and extreme wall-normal velocity fluctuations in near-wall turbulence. Physics of Fluids 24:035110

Lynch K and Scarano F (2013) A high-order time-accurate interrogation method for time-resolved PIV. Measurement Science and Technology 24:035305

Novara M, Schanz D, Geisler R, Gesemann S, Voss C, and Schröder A (2019) Multi-exposed recordings for 3D Lagrangian particle tracking with multi-pulse Shake-The-Box. Experiments in Fluids 60:44-63

Schanz D, Gesemann S, and Schröder A (2016) Shake-The-Box: Lagrangian particle tracking at high particle image densities. Experiments in Fluids 57:1-27

Schröder A, Schanz D, Novara M, Philipp F, Geisler R, Agocs J, Knopp T, Schroll M, and Willert CE (2018) Investigation of a high Reynolds number turbulent boundary layer flow with adverse pressure gradients using PIV and 2d- and 3d- Shake-The-Box. in 19th International Symposium on Applications of Lasers and Imaging Techniques to Fluid Mechanics. Lisbon, Portugal 
Wieneke B (2012) Iterative reconstruction of volumetric particle distribution. Measurement Science and Technology 24:024008

Willert C, Cuvier C, Foucaut J, Klinner J, Stanislas M, Laval J, Srinath S, Soria J, Amili O, Atkinson C, Kähler C, Scharnowski S, Hain R, Schröder A, Geisler R, Agocs J, and Röse A (2018a) Experimental evidence of near-wall reverse flow events in a zero pressure gradient turbulent boundary layer. Experimental Thermal and Fluid Science 91:320 - 328

Willert C, Soria J, Cuvier C, Foucaut J, and Laval J (2018b) Flow reversal in turbulent boundary layers with varying pressure gradients. in 19th Intern. Symp. on Applic. of Laser and Imaging Techniques to Fluid Mecahnics. Lisbon, Portugal
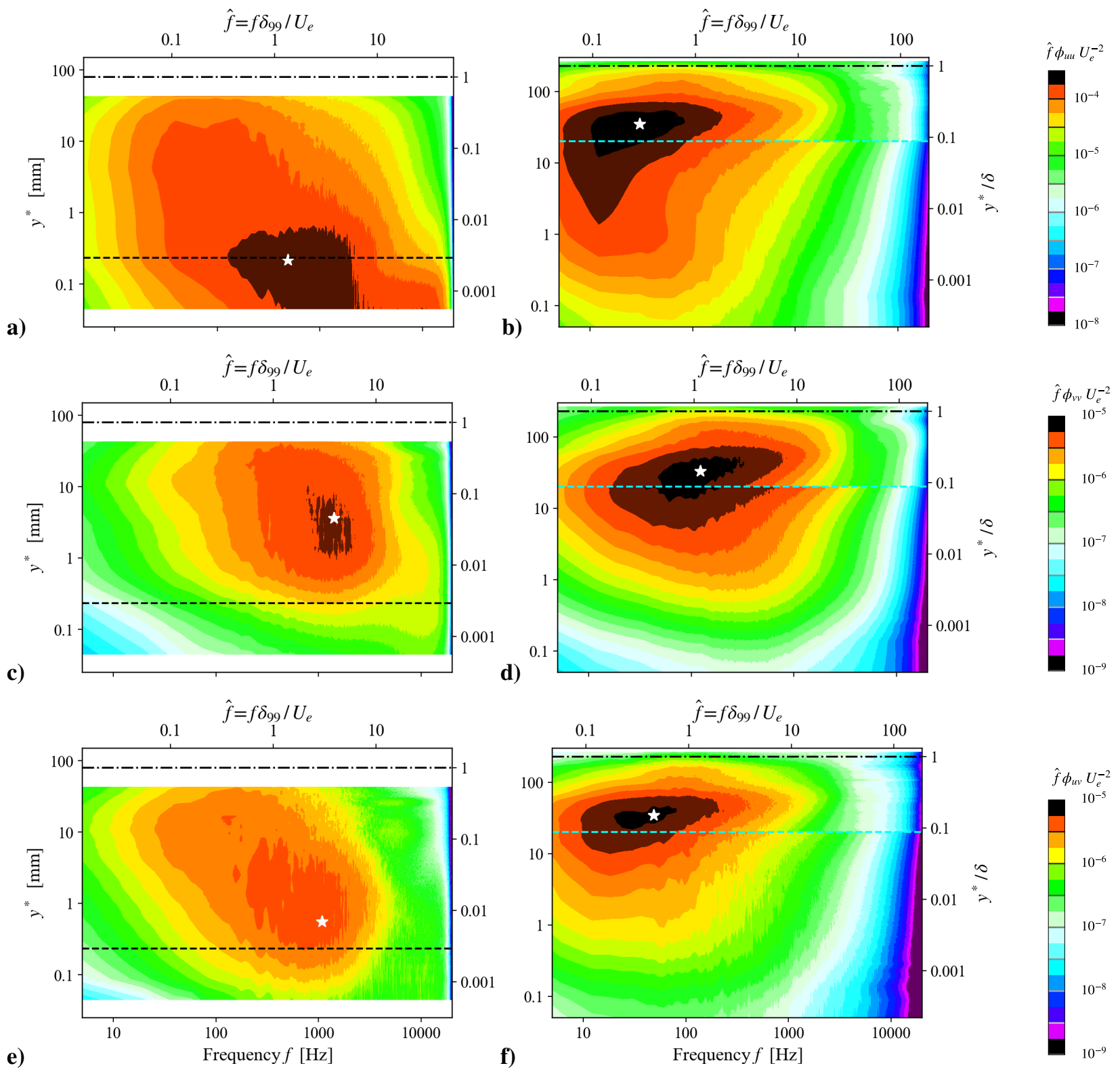

Figure 9: Pre-multiplied power spectra obtained for the respective velocity components for $U_{\infty}=29.0 \mathrm{~m} / \mathrm{s}$ for ZPG (left column) and APG conditions (right column); spectrum of streamwise velocity $\left\langle u^{\prime} u^{\prime}\right\rangle$ (a,b), wall-normal velocity $\left\langle v^{\prime} v^{\prime}\right\rangle$ (c,d) and Reynolds stress $\left\langle u^{\prime} v^{\prime}\right\rangle$ (e,f). Data is normalized by the respective edge velocity $U_{e}$ and boundary layer thickness $\delta_{99}$. Dashed black line in left plots indicates the location of the inner turbulence peak at $\left(y^{+}=15\right)$. Dashed light-blue line in right plots indicates a chosen boundary at $y=20 \mathrm{~mm}$ between camera view 1 and view 2 . 